\title{
Inspiré par les lipides (Médaille Chevreul 2014)^
}

\author{
John L. Harwood ${ }^{\star \star}$, Traduction de Valérie Duflot et Philippe Guesnet, $O C L$ journal \\ School of Biosciences, Cardiff University, Wales, UK
}

Reçu le 20 décembre 2014 - Accepté le 12 janvier 2015

\begin{abstract}
Résumé - En tant qu'étudiant en biochimie médicale, je n'étais pas certain de ce que je souhaitais faire de ma vie une fois diplômé. Puis, un jour, j'ai assisté à une présentation sur les lipides membranaires. Il s'avérait qu'ils étaient loin d'être les composés structurels inertes que l'on décrivait souvent, mais qu'ils se renouvelaient très rapidement. Une classe de lipides - les inositides (PtdIns4P, PtdIns $(4,5)$ P2) - possède ainsi une demi-vie de quelques minutes ! Une découverte qui me fascina et m'inspira non seulement lors de mon parcours post-doctoral sur les inositides avec J.N. (Tim) Hawthorne mais également pour entamer et poursuivre mes recherches dans le domaine des lipides. Je n'ai aucun regret pour ce qui a été un fascinant voyage. Le grand scientifique français Chevreul a contribué à des avancées dans de nombreux domaines scientifiques; c'est avec humilité que j'aborderai l'un d'entre eux, les lipides, à travers divers points sur lesquels j'ai pu travailler.
\end{abstract}

Mots clés : Acide $\alpha$-linolénique / inositides / tensioactif pulmonaire / AGPI / métabolisme sulpholipidique / stress et adapatation à la température

\begin{abstract}
Inspired by lipids (the Chevreul Award Lecture 2014). As an undergraduate student of Medical Biochemistry, I was unsure what I wanted to do with my life after graduating. Then, one day, I had a lecture about membrane lipids. It turned out that not only were they far from the inert structural compounds that they were often portrayed to be but they turned over quite rapidly. But there was one class of lipids - the higher inositides (PtdIns4P, PtdIns $(4,5) \mathrm{P}_{2}$ ) that had a T1/2 of only a few minutes! I found this fascinating and was inspired not only to do my post-graduate work with J. N. (Tim) Hawthorne on inositides but to continue work on lipids ever since. I have no regrets for it has been a fascinating journey. The great French scientist Chevreul made contributions to several different areas of science so, in humble appreciation of his seminal discoveries, I will discuss some diverse lipid topics that I have worked on.
\end{abstract}

Keywords: $\alpha$-Linolenic acid / inositides / pulmonary surfactant / PUFA / sulpholipid metabolism / temperature stress and adaptation

\section{Une découverte inattendue}

Ma première expérience de chercheur post-doctoral se déroula à l'Université de Californie, à Davus, sous la direction du Professeur Paul Stumpf. Ce fut ma première incursion dans le monde des plantes, à propos desquelles je n'avais pas eu la moindre formation scientifique ! J'ai commencé mon travail de recherche sur la synthèse des acides gras dans la germination du pois et fut rapidement immergé dans le sujet avec nombre de résultats particulièrement intéressants et originaux. Un jour, je décidai de recourir à quelques inhibiteurs pour modérer l'approvisionnement en substrat de la voie de synthèse des acides gras. L'un deux était l'arsenic, un inhibiteur de deux réactions

\footnotetext{
* Traduit de la version anglaise publiée dans EJLST : Inspired by lipids (the Chevreul Award Lecture 2014). Eur. J. Lipid Sci. Technol. 116: 1259-1267. Copyright Wiley-VCH Verlag GmbH \& Co. KGaA. Reproduced with permission.

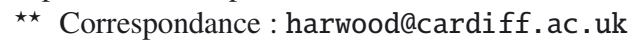

de déshydrogénation/décarboxylation du cycle de Krebs. À ma surprise, cela élimina le radiomarquage de tous les acides gras plus longs que le palmitate (Harwood et Stumpf, 1971). Ces données expérimentales permirent d'identifier une nouvelle enzyme encore inconnue à l'époque, la ß-kétoacyl-ACP synthase II (KAS II), impliquée dans l'étape d'allongement de chaîne de palmitate en stéarate (condensation) et sensible à ces inhibiteurs (Harwood et Stumpf, 1972). Plus tard, mon laboratoire travailla sur une troisième enzyme de condensation, KAS III, découverte chez Escherichia coli (Jackowski et Rock, 1987), et également exprimée dans les végétaux (Walsh et al., 1990 ; Jones et al., 2003).

\section{Le surfactant pulmonaire}

Je fus ensuite nommé enseignant chercheur à l'Université de Cardiff, une ville connue pour ses mines de charbon. 


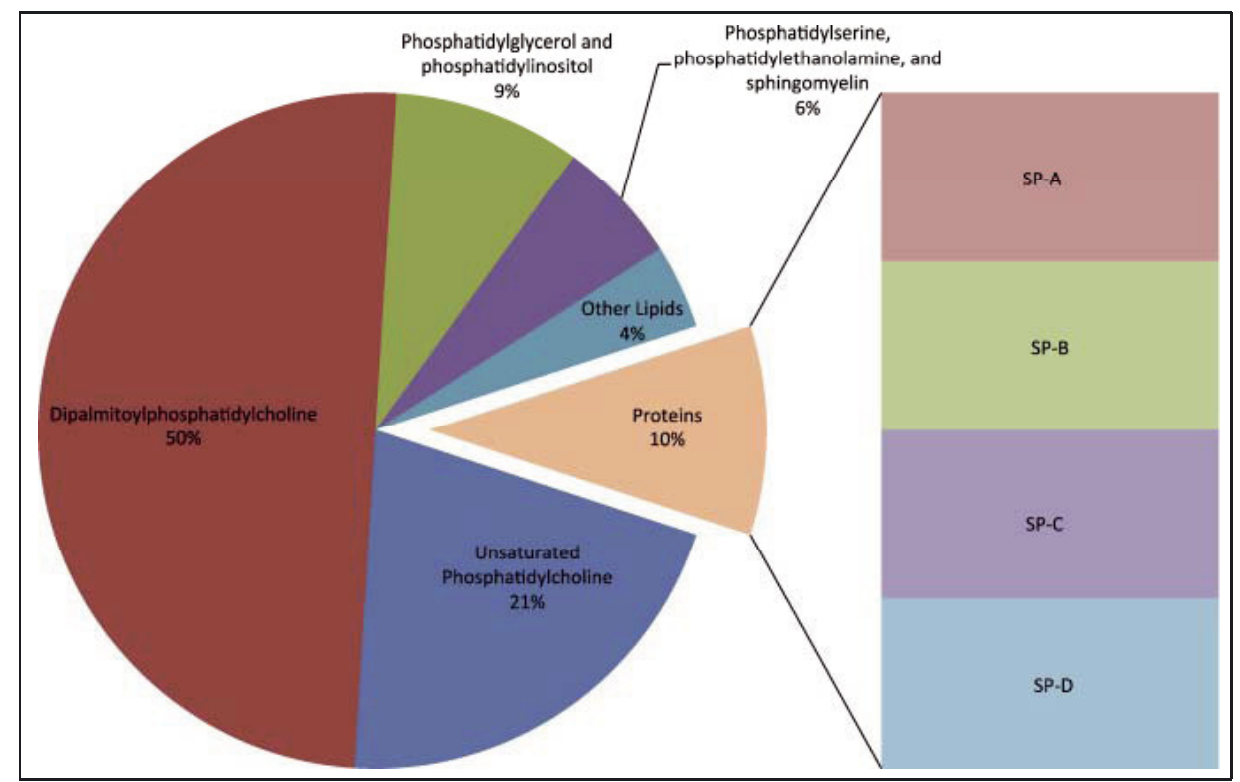

Fig. 1. Composition typique des surfactants alvéolaires. Globalement, les tensio-actifs sont composés à 85-91 \% de lipides sous la forme majoritairement de phosphatidyl-choline (PtdCho), le reste étant constitué par quatre protéines dénommées SP-A, SP-B, SP-C et SP-D. La PtdCho est le composant majoritaire, l'espèce moléculaire dipalmitoyl-PtdCho représentant à elle seule la moitié du total. D'après Agassandian et Mallampalli (2013), Biochim. Biophys. Acta 1831: 612-625 (avec leur aimable autorisation).

L'industrie du charbon étant à l'origine de maladies respiratoires, j’ai commencé à considérer cette problématique à travers le prisme de la biochimie lipidique.

Lorsque nous expirons, nos poumons sont gardés en partie gonflés pour que l'inspiration suivante soit plus aisée. Cette propriété est assurée par la sécrétion de tensio-actif pulmonaire par les cellules épithéliales alvéolaires de type II (Robertson et al., 1984), dont la détérioration entraîne une détresse respiratoire. L'importance physiologique de ce tensio-actif a été particulièrement bien démontrée chez l'enfant prématuré souffrant de détresse respiratoire dont la mortinatalité était particulièrement élevée. En effet, sa synthèse a lieu exclusivement durant les six dernières semaines de gestation.

Nous avons ainsi purifié le tensio-actif pulmonaire d'un certain nombre de mammifères (Harwood, 1975) et, avec les connaissances déjà acquises sur les humains (Clements, 1997), avons pu définir les caractéristiques biochimiques uniques de ce remarquable mélange de lipoprotéines (Fig. 1). Grâce à ces connaissances fondamentales, nous avons pu concevoir un tensio-actif artificiel (ALEC) qui, avec d'autres préparations de tensio-actifs, pouvaient être délivrés par voie respiratoire aux nourrissons souffrant de «détresse respiratoire aiguë» (Wrobel, 2004). L'instillation de tels tensioactifs, en complément d'une hyperventilation assistée, ont représenté une telle réussite médicale qu'il n'y a pratiquement plus de nos jours de mortinatalité par détresse respiratoire chez le nourrisson prématuré.

\section{L' $\alpha$-linolénique, l'acide gras le plus abondant sur terre}

Les feuilles des plantes vertes contiennent des quantités remarquables d'acide alpha-linolénique - environ $65 \%$ de leurs acides gras totaux, acide gras polyinsaturé (AGPI) qui s'avère particulièrement concentré dans les membranes des chloroplastes. Étonnamment, ce n'est qu' autour des années 1980 que la voie de synthèse de novo de cet AGPI a été caractérisée.

Après quelques études réalisées sur Chlorella vulgaris au sein du laboratoire d'Anthony James dans le centre de recherche d'Unilever de Sharnbrook (Gurr et al., 1969; et al., 1967), il apparut que l'acide alpha-linolénique pouvait être néo-synthétisé à partir de substrats lipidiques complexes. Tandis que Slack, Roughan et leur groupe travaillaient sur la désaturation du linoléate au cours du développement de graines oléagineuses (Slack et al., 1979), nous avons plutôt opté d'aborder cette thématique au niveau des feuilles. Les premiers chercheurs ont considéré la PtdCho (phosphatidylcholine) comme le substrat de cette voie de synthèse, alors que nous avons envisagé que le monogalactosyldiacylglycérol (MGDG) était utilisé préférentiellement (Wharfe et Harwood, 1978). Ces vues divergentes furent réconciliées quand il fut démontré qu'une fatty acid désaturase (fad)3 située au niveau du réticulum endoplasmique utilisait la PtdCho comme substrat, tandis que l'action des fad7- et fad8-désaturases formait de l'alpha-linolénique à partir du MGDG dans le chloroplaste (Wallis et Browse, 2002). En même temps nos données servirent de support pour comprendre les processus de coopération existant entre le réticulum endoplasmique et le plaste dans la fourniture globale en acides gras foliaires (Tab. 1 et Fig. 2).

\section{Métabolisme sulpholipidique}

Les thylakoïdes des algues, des cyanobactéries et des plantes possèdent une composition en lipides unique comparée aux autres membranes des cellules. En effet, ils sont 


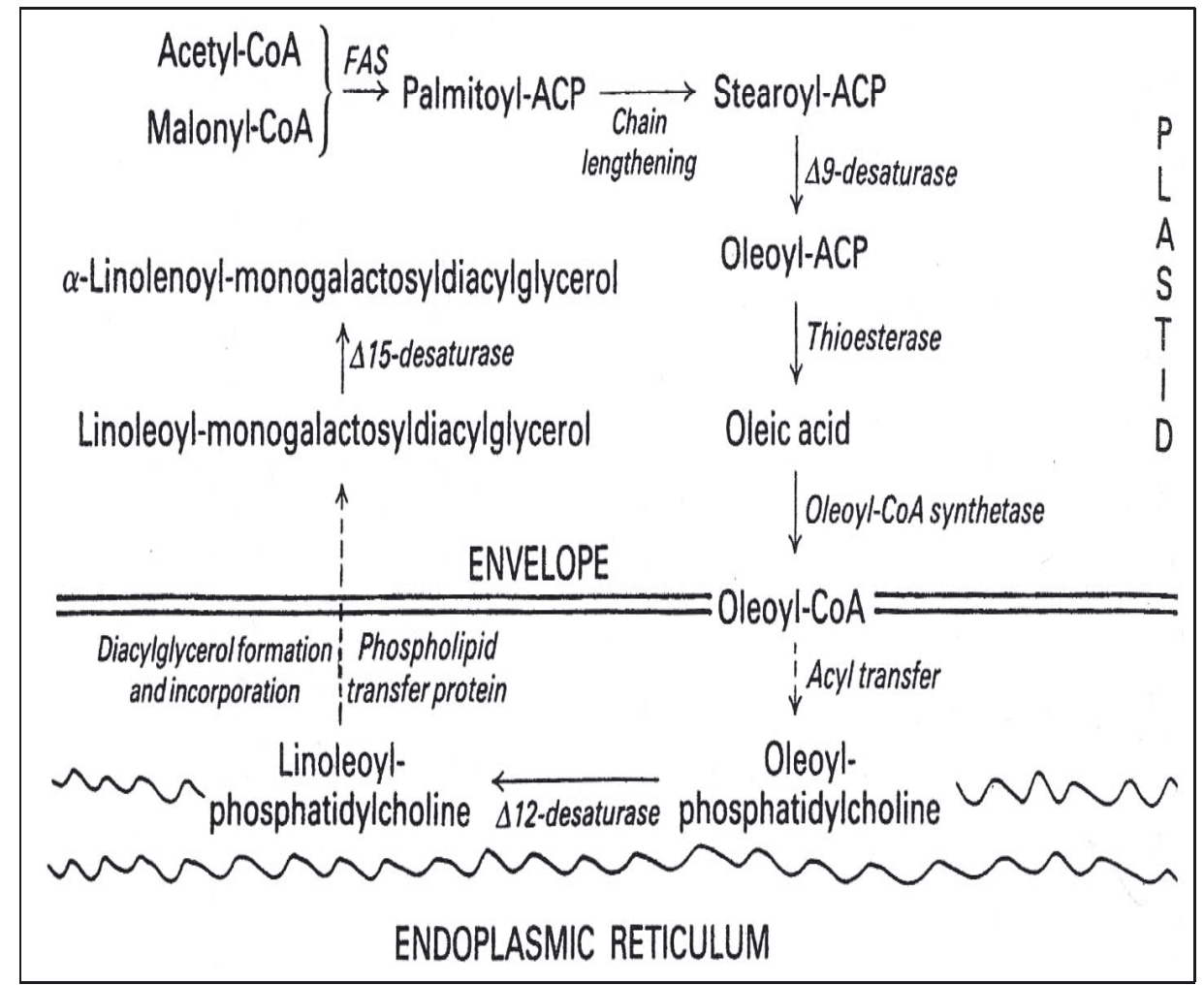

Fig. 2. Description simplifiée de la voie de formation globale d' $\alpha$-linolénate dans les feuilles des plantes utilisant la «voie eukaryote» de la synthèse lipidique. Notez que la formation de linolénate par la $\Delta 12$-désaturase et d' $\alpha$-linolénate par la $\Delta 15$-désaturase est associée à la membrane tandis que les réactions conduisant à l'oléate sont toutes solubles (c'est-à-dire dans le stroma des plastes). Reproduit avec l'aimable autorisation de Blackwells Scientific. From Lipid Biochemistry. 5th ed. Blackwells Scientific, Oxford, U.K. (2002).

Tableau 1. Radioactivités spécifiques des acides gras des glycérolipides majeurs de la feuille.

\begin{tabular}{cccccc}
\hline & & $16: 0$ & $18: 1$ & $18: 2$ & $18: 3$ \\
\hline MGDG & $1,5 \mathrm{~h}$ & 2,7 & 14,3 & 3 & 0,3 \\
DGDG & - & 1,9 & 15,1 & 2,7 & n.d. \\
PC & - & 3,9 & 15,3 & 3,2 & n.d. \\
MGDG & $5,5 \mathrm{~h}$ & 5,4 & 45,4 & 10,7 & 1,7 \\
DGDG & - & 2,3 & 43 & 11,4 & 1,4 \\
PC & - & 6,7 & 46,4 & 13,2 & n.d. \\
\hline
\end{tabular}

MGDG, monogalactosyldiacylglycerol ; DGDG, digalactosyldiacylglycerol ; PC, phosphatidylcholine. Les données montrent un équilibre de l'oléate et du linoléate entre PC et les deux galactoglycérides, alors que l' $\alpha$-linolénate est formé à partir du MGDG. Données issues de Wharfe et Harwood, 1978.

constitués à $90 \%$ de deux lipides complexes, un phosphoglycéride, le phosphatidylglycérol, et les glycosylglycérides (Tab. 2). Au moment où nous nous sommes intéressés au métabolisme lipidique du thylakoïde et à sa fonction, le sulphoquinovosyldiacylglycerol (SQDG, le sulpholipide des plantes) était encore peu connu. En ce qui concerne sa synthèse, les hypothèses proposées étaient pratiquement plus nombreuses que les preuves expérimentales. Les premières hypothèses avancées sont récapitulées dans la publication d'Harwood (1980). Un point de désaccord portait sur la formation du groupe sulphonate. Une suggestion de la chimie, à savoir l'addition de sulphite à un glucose-enide (Lehmann et Benson, 1964), couplé à la connaissance d'une séquence de réactions donnant à partir de l'UDP-galactose un glucose-enide (Barber, 1963), nous ont conduit à mener une série d'expériences biochimiques, qui examinèrent plusieurs parties de la voie globale que nous fûmes en mesure de proposer (Pugh et al., 1995) (Fig. 3). L'isolement de gènes codant pour les enzymes impliquées par le groupe de Benning confirma cette voie métabolique (Shimijima, 2011).

Cependant, avait été laissée de côté la question de la répartition du SQDG. Puisque le SQDG est un composant significatif du cycle du soufre, il est important de comprendre et définir son catabolisme. De précédents travaux avaient montré l'activité d'acylhydrolases végétales (Burns et al., 1977), qui détachaient les deux acides gras de leur squelette glycérol, suivie par la libération de sulphoquinovose (SQV). Ces activités permettent aux plantes de produire du SQV lors du flétrissement de la végétation, mais un catabolisme plus poussé repose sur les micro-organismes du sol.

En raison d'une comparaison évidente des dérivés de glucide sulphonés avec les intermédiaires phosphorylés de la glycolyse, Benson a suggéré une « voie sulphoglycolytique» (Lee et Benson, 1964) et identifié un sulphoacétate intermédiaire dans un Flavobacterium du sol (Martelli et Benson, 1964). Nous avons prolongé l'étude en isolant diverses bactéries du sol (comme Klebsiella et Agrobacterium), qui pouvaient utiliser un sulphoquinovose comme source unique de carbone et 
John L. Harwood, Traduction de Valérie Duflot et Philippe Guesnet, OCL journal : OCL 2015, 22(2) A202

Tableau 2. La nature unique des lipides des thylakoides.

\begin{tabular}{|c|c|c|c|c|c|c|}
\hline Lipides (\% en poids) & E. coli* ${ }^{*}$ & Levure $^{1}$ & Cyanobactérie $^{2}$ & Chloroplaste $^{3}$ & Erythocyte $^{4}$ & Mitochondrie $^{5}$ \\
\hline PtdCho & - & 39 & - & tr. & 32 & 41 \\
\hline PtdEtn & 82 & 30 & - & tr. & 15 & 34 \\
\hline PtdGro & 6 & 1 & 17 & 12 & & \\
\hline PtdSer & - & 5 & - & & 7 & 1 \\
\hline \multicolumn{7}{|l|}{ Glycosylglycerides } \\
\hline MGDG & & & 54 & 41 & - & - \\
\hline DGDG & & & 17 & 23 & - & - \\
\hline SQDG & & & 11 & 16 & - & - \\
\hline Stérols & - & 3 & - & tr. & 25 & tr. \\
\hline Sphingolipides & - & tr. & - & - & 6 & 2 \\
\hline
\end{tabular}

$* 12 \%$ Diphosphatidylglycérol (cardiolipine). ${ }^{1}$ : S. cerevisiae,${ }^{2}$ : Anabaena variabilis,${ }^{3}$ : thylakoïdes de chloroplaste (valeur moyenne), ${ }^{4}$ : rat,

5 : foie de rat.

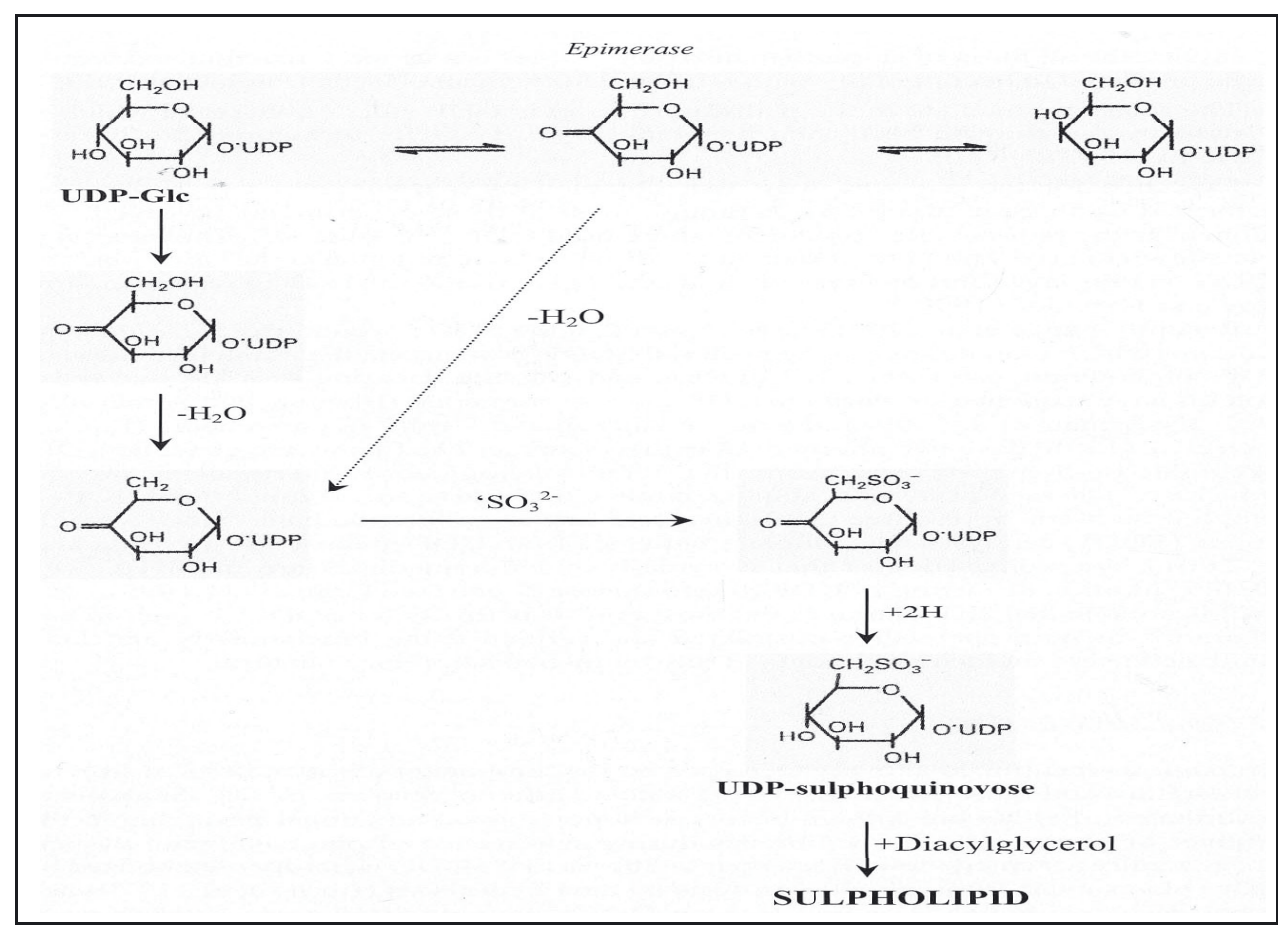

Fig. 3. Voie métabolique pour la biosynthèse du sulphoquinovosyl diacylglycérol (sulpholipide des plantes) Pour plus de détails, voir (Pugh et al., 1995).

d'énergie pour leur croissance (Roy et al., 2003). Nous avons démontré clairement l'utilisation d'une séquence glycolytique via le sulpholactate et le sulphopropanédiol (Fig. 4).

Récemment, une publication a confirmé les trois premières réactions de production du sulpholactaldéhyde chez E. coli K-12. Chez cette bactérie, une étape supplémentaire de réduction s'effectue et le produit formé, le 2,3-dihydroxypropane-1sulphonate, pourrait être excrété au moyen d'une protéine de transport spécifique (Denger et al., 2014). Toutes les protéines impliquées dans cette voie étaient codées dans un groupe de dix gènes chez E. coli. Les auteurs ont mis en évidence l'importance du SQV dans le cycle biogéochimique du soufre et aussi les capacités variables de micro-organismes différents pour utiliser une voie «sulphoglycolytique» (Denger et al., 2014).

\section{Les poïkilothermes à la merci du changement climatique}

Pendant plusieurs décennies, nous avons travaillé sur le stress de la température (et l'adaptation) chez différents organismes. Pour les animaux poïkilothermes (à « sang froid », soit la grande majorité des organismes), cela peut être un problème réel, renforcé par le changement climatique. Afin de mieux comprendre le processus moléculaire d'adaptation dans sa globalité, nous avons choisi de travailler avec un protozoaire commun du sol, Acanthamoeba castellanii (Fenchel, 1984).

Cet organisme grandit entre 5 et $32^{\circ} \mathrm{C}$ et se nourrit de bactéries de sol (Fenchel, 1982). Nous avons examiné les changements des lipides membranaires chez Acanthamoeba en faisant varier la température de croissance de 30 à $15{ }^{\circ} \mathrm{C}$. À notre 


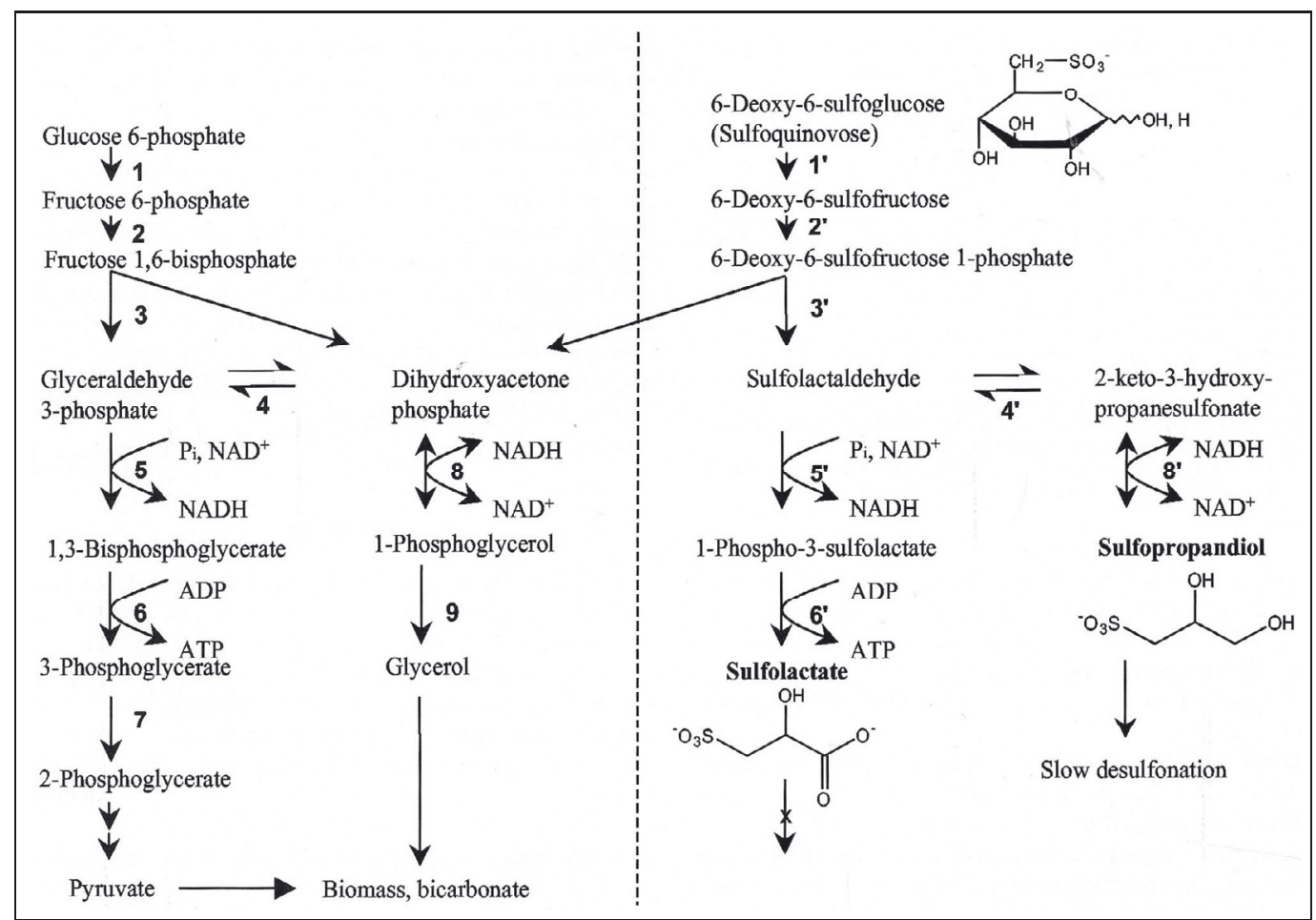

Fig. 4. Catabolisme du groupe sulphoquinovose du sulpholipide de la plante par les bactéries du sol. Pour plus de détails voir Roy et al., 2003.

Tableau 3. Séquence d'évènements lorsque Acanthamoeba s'adapte à des températures de croissance basses.

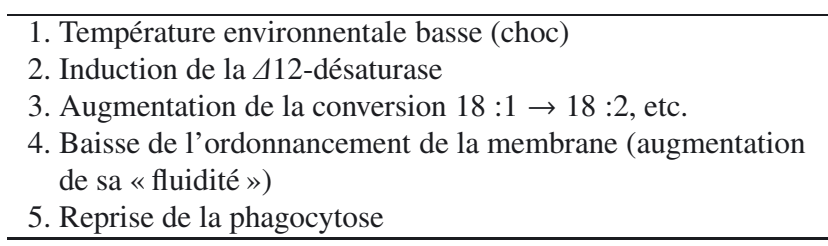

surprise, nous avons constaté que le principal changement au niveau du métabolisme des lipides était une augmentation de la conversion de l'oléate en linoléate. Bien qu'une augmentation de l'insaturation soit une réponse commune à la baisse de la température (afin de maintenir la fluidité de membrane), nous avons été étonné d'observer des variations de synthèse d'acides gras insaturés essentiels (linoléate) chez un animal (protiste).

Les concentrations en certains acides gras polyinsaturés à plus longue chaîne, y compris l'acide eicosapentaénoïque (EPA), ont également augmenté (Fig. 5) (Jones et al., 1991). La nature inhabituelle de cette adaptation nous a poussé à étudier la désaturase concernée (Jones et al., 1993) sur la durée entière de la chronologie menant de l'induction d'enzyme à la modification de la fluidité membranaire (Avery et al., 1995b) et la reprise de la phagocytose (Avery et al., 1995a) (Tab. 3).

L'isolement du gène 1 de la $\Delta 12$-désaturase par nos collègues de Rothamsted (Sayanova et al., 2006) a conduit à un travail commun au cours duquel le gène a été exprimé dans la levure, ce qui a abouti à la fois à des $\Delta 12$ - et $\Delta 15$-désaturations d'acides gras à 16 et 18 atomes de carbone. Dans le cas du

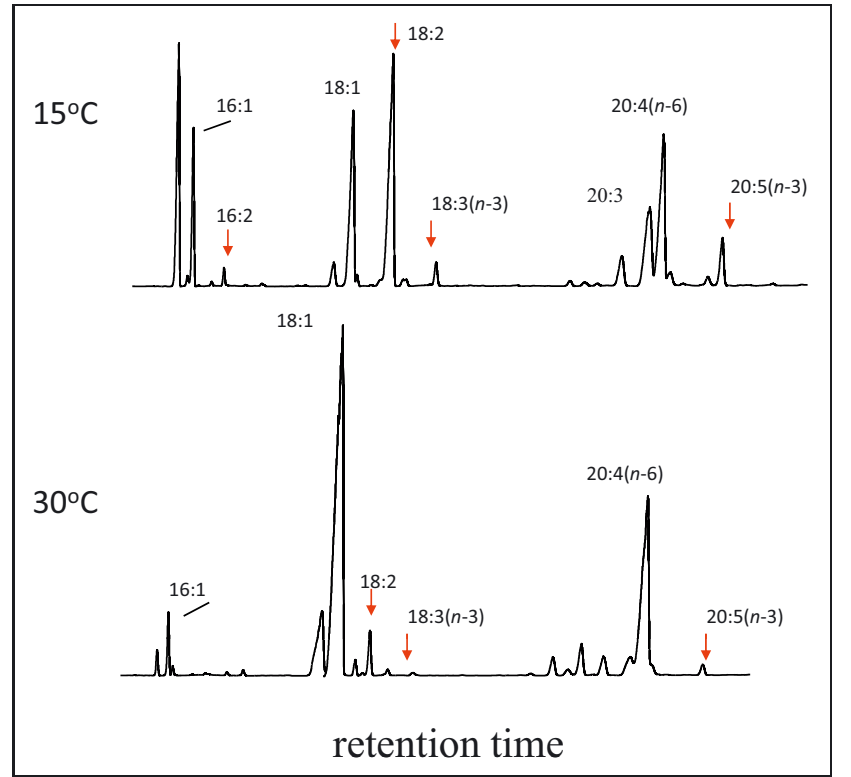

Fig. 5. Des températures plus basses lors de la croissance de Acanthamoeba castellanii augmentent sa concentration en acides gras polyinsaturés. Notez que le linoléate et l' $\alpha$-linolénate sont augmentés, soulignant à la fois une activation des étapes de $\Delta 12$ - et $\Delta 15$-désaturation. Voir Jones et al., 1991.

hexadécatrienoate, l'acide gras produit contenait un méthyle terminal à double liaison (Sayanova et al., 2006), une position plutôt inhabituelle, qui a été rarement rapportée (Dayan et al., 2003). Ainsi, la désaturase d'Acanthamoeba était bifonctionnelle et, en fait, par analogie avec une désaturase 


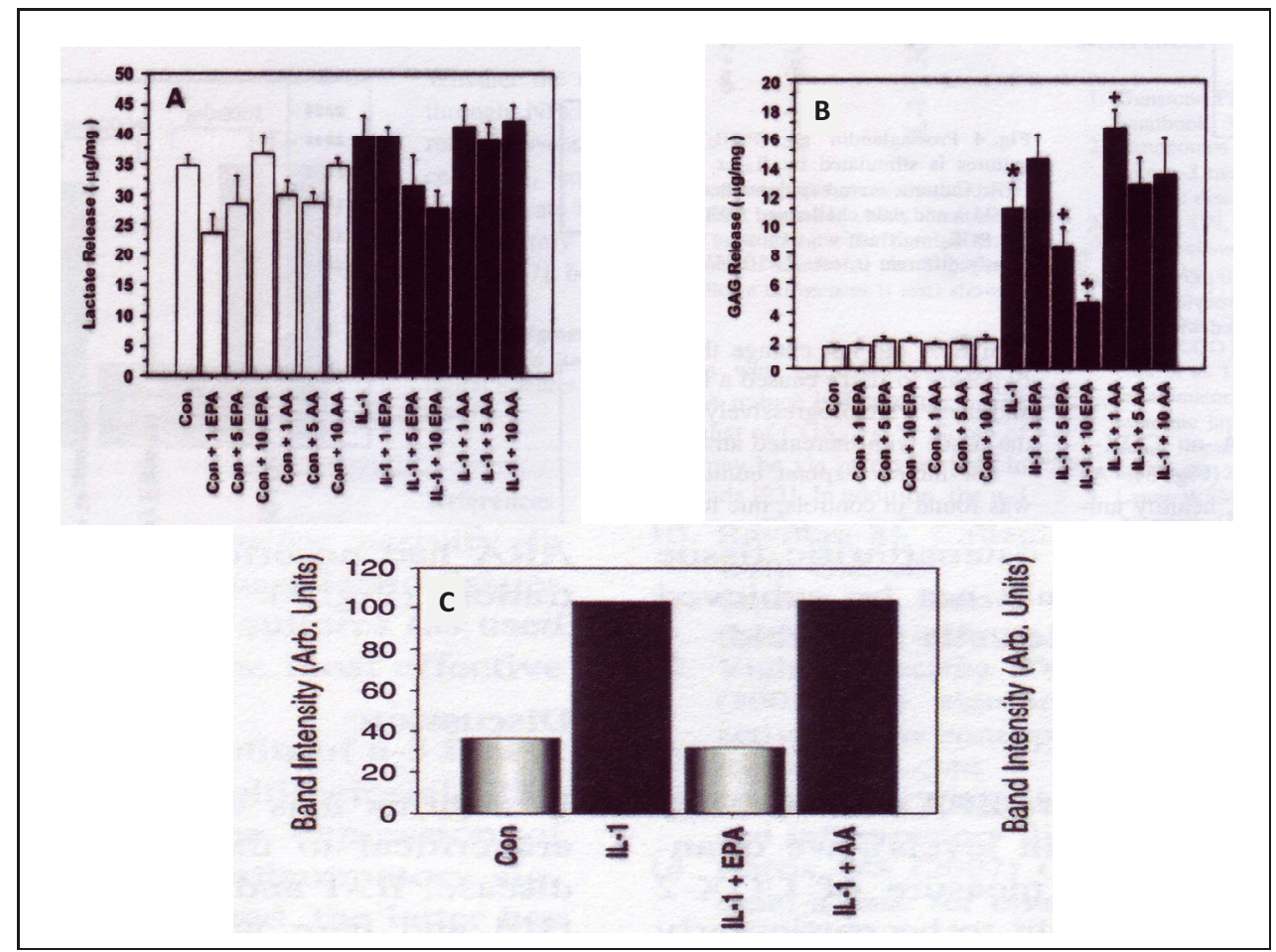

Fig. 6. Effets contrastés des acides gras polyinsaturés n-3 (EPA) et n-6 (acide arachidonique, AA) sur la dégradation du cartilage et l'expression de la cyclo-oxygénase. Sur (A), est montrée la libération de lactate (une mesure de viabilité cellulaire) et sur (B) la libération de glycosaminoglycane (GAG) (une mesure de dégradation de cartilage) de cultures d'explants de cartilages bovins. Sur (C) est montré l'effet de l'interleukine-1 et des AGPI sur les niveaux d'ARNm de la cyclo-oxygénase 2. Données issues de Hurst et al., 2009, avec leur aimable autorisation.

très semblable de Mortierella, qui a été récemment rapportée (Kikukawa et al., 2013), pourrait même être tri-fonctionnelle.

\section{Bénéfices santé des AGPI n-3}

Les acides gras polyinsaturés alimentaires de la série n-3 sont considérés comme bénéfiques vis-à-vis d'un certain nombre de pathologies inflammatoires chroniques y compris l'arthrite, les maladies cardiovasculaires, de certains types de cancer et de la fonction neurologique (Harwood et Caterson, 2006; Watson, 2009).

On considère que l'arthrite affecte plus de $10 \%$ de la population mondiale âgée de plus de 60 ans, causant une souffrance immense aux patients. Au Royaume-Uni, le coût de santé représente actuellement de l'ordre de 1,1 milliard de livres par an (1,39 milliard d'euros). Une fois que l'ostéo-arthrite sévère se développe, la chirurgie s'avère le seul recours médical. Cependant, il peut être possible d'aider à prévenir (mais aussi à soulager les symptômes) via un régime alimentaire riche en acides gras polyinsaturés (AGPI) à longue chaîne n-3 (EPA et/ou acide docosahexaénoïque, DHA). En raison des résultats encourageants auprès de patients (Calder et Zurier, 2001), nous avons cherché à décrypter les mécanismes moléculaires par lesquels un tel neutraceutique pouvait être efficace.

Nous avons utilisé un modèle très bien caractérisé d'explants de tissu conjonctif issus de bovins ou de porcs, ou bien encore de tissu conjonctif humain prélevé sur des personnes subissant une chirurgie de remplacement du genou.
Nous avons mesuré la répartition du tissu conjonctif, la production de cytokines et l'expression et l'activité de cyclooxygénases (COXs) après exposition à divers AGPI n-3 et n-6. La destruction de cartilage par les protéases (mesurée par les glycosaminoglycanes) et la sécrétion d'une variété de cytokines $(1 \mathrm{~L}-1, \mathrm{TNF}-\alpha$, etc.) ont été toutes les deux réduites à des niveaux indétectables en présence d'EPA et de DHA, partiellement en présence d'acide $\alpha$-linolénique, mais aucunement avec un AGPI n-6 à longue chaîne l'acide arachidonique (Curtis et al., 2002 ; Zainal et al., 2009) (Fig. 6). De façon intéressante, les AGPI n-3 réduisent l'activité de COX-2 mais pas celle de COX-1 (Zainal et al., 2009; Hurst et al., 2009). De façon remarquable dans les situations inflammatoires, c'est par ailleurs COX-2 qu'il est important d'inhiber spécifiquement plutôt que COX-1. Ces données encourageantes ont ouvert la voie à une collaboration avec un fabricant d'aliments pour animaux domestiques durant laquelle nous avons montré qu'un supplément alimentaire destiné aux chiens souffrant d'arthrite administré pendant seulement un mois induisait une réduction de la boiterie, de la rigidité, etc. ${ }^{1}$. Les AGPI n-3 représentent désormais un complément classique dans un large échantillon de produits alimentaires canins.

La démence, dont la maladie d'Alzheimer représente $70 \%$ des cas, s'avère encore plus répandue que l'arthrite. Plusieurs grandes études épidémiologiques ont montré que la consommation d'AGPI n-3 peut réduire le risque de développer la

1 U.S. Patent application 11/0577 18, (filed Feb.14, 2005) Method for decreasing cartilage damage in dogs. 
maladie d'Alzheimer de plus de $60 \%$ (Kalmijn et al., 1997; Bargerger-Gateau et al., 2002; Morris et al., 2003). Nous avons utilisé le modèle de souris Tg2576, qui a été largement utilisé pour de telles études. Il exprime la mutation de la protéine précurseur amyloïde humaine et commence à montrer une détérioration des fonctions cognitives et à accumuler des dépôts amyloïdes à l'âge de 8 mois environ (Elder et al., 2010).

Un régime alimentaire enrichi en DHA a montré que son incorporation dans des lipides cérébraux était sélective. Par exemple, parmi les deux phosphoglycérides qui contiennent les plus fortes proportions de DHA, à savoir la phosphatidyléthanolamine (PtdEtn) et la phosphatidyl-sérine (PtdSer), seule la PtdEtn a été significativement affectée par la supplémentation en DHA (Fig. 7). En général, les augmentations du contenu en DHA des lipides dans le cortex, le cervelet, ou les régions de l'hippocampe ont été accompagnées par une diminution réciproque de l'AGPI n-6 arachidonate et des acides docosatetraénoïques. Ces données soulignèrent l'existence de mécanismes de régulation sophistiqués du métabolisme lipidique cérébral.

Des études comportementales ont été effectuées pour tester l'anxiété (test d'enfouissement dit «marble burying», test « elevated maze ») et les divers types de cognition (test alternatif dans un labyrinthe en $T$, etc.). Ceux-ci ont montré qu'une supplémentation alimentaire en DHA pouvait avoir des effets positifs sur le comportement (par exemple, Falinska et al., 2012) et sont concordants avec de nombreuses autres études (McCann et Ames, 2005) montrant l'efficacité des AGPI n-3. Sur le plan nutritionnel, il est indispensable que ces AGPI n-3 soient consommés quotidiennement aux niveaux recommandés par les diverses organisations de nutrition (par exemple, American Heart Association, l'Organisation mondiale de la Santé). Une très bonne et récente revue pour couvrir les besoins en AGPI n-3 via un régime alimentaire adéquat en ces acides gras avec un ratio $\mathrm{n}-6 / \mathrm{n}-3$ équilibré (autour 4 ) vient d'être publiée (Lands, 2014).

\section{Approvisionner le monde avec graisses et huiles de composition adéquate}

Pendant les cinq dernières décennies, la consommation et l'utilisation des graisses et des huiles a augmenté de plus de $5 \%$ chaque année. Avec des populations croissantes et un besoin de fournir à l'industrie des ressources renouvelables pour la production de produits chimiques, de telles tendances vont probablement s'accélérer. De l'ordre de $70 \%$ de ces approvisionnements proviennent de seulement quatre cultures oléagineuses : par ordre décroissant d'importance le palme, le soja, le colza (canola) et le tournesol (Gunstone et al., 2007). Deux aspects du métabolisme et stockage de l'huile dans la graine oléagineuse se doivent d'être étudiés : la qualité d'une part et la quantité. Nous nous sommes concentrés particulièrement sur le second point.

Il y a diverses manières d'obtenir des informations sur la régulation de l'accumulation d'huile dans la graine oléagineuse (voir Harwood et al., 2013). Parmi ces méthodes, nous avons utilisé l'analyse de contrôle pour obtenir des informations quantitatives. La théorie qui se cache derrière ces deux

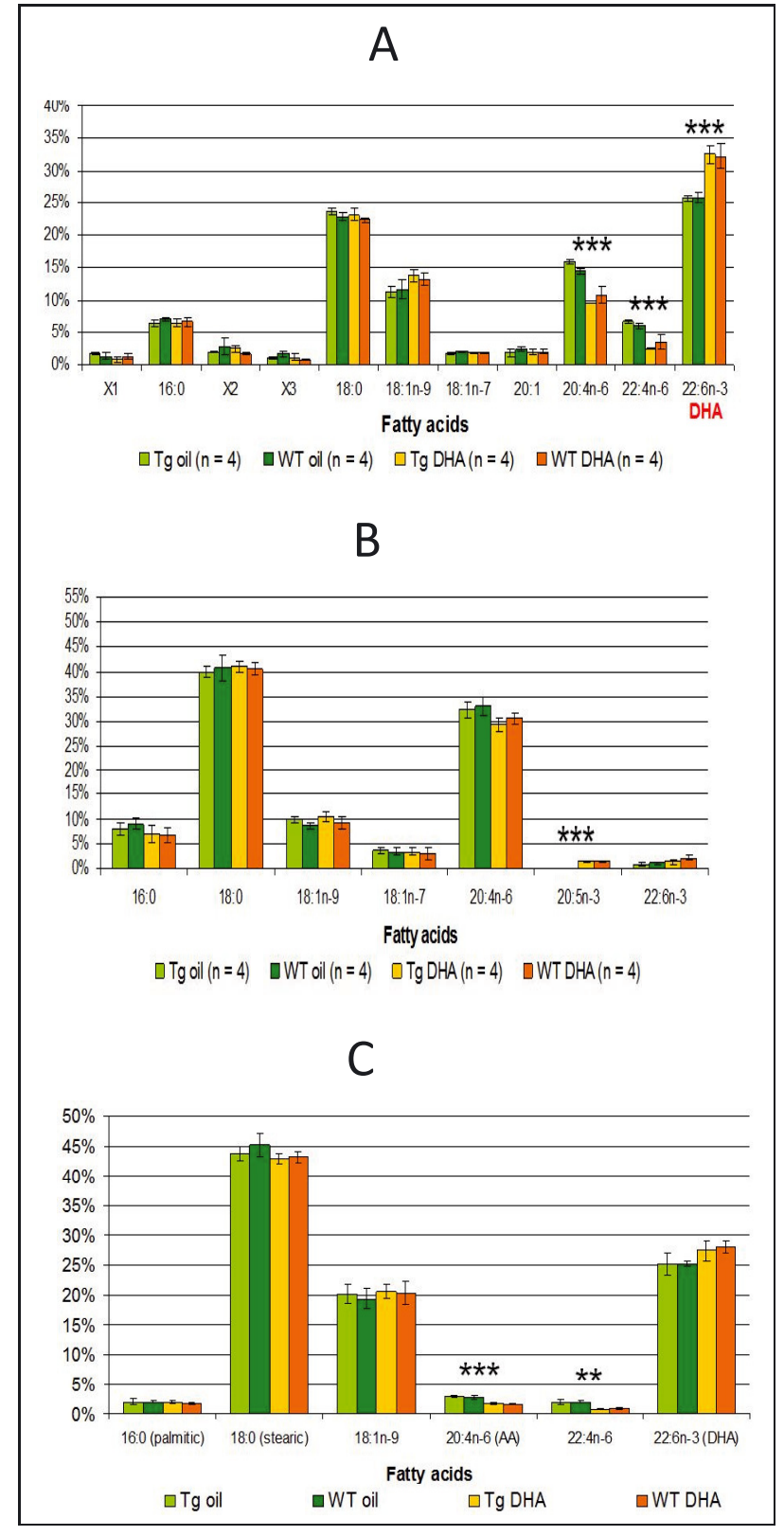

Fig. 7. Les phosphoglycérides du cerveau possèdent chacun une composition en acides gras spécifique qui peut être modifiée par le régime alimentaire à des degrés différents. Le contenu en acide gras de phosphoglycérides individuels issus de l'hippocampe du cerveau de souris est représenté. $\mathrm{A}=$ PtdEtn, $\mathrm{B}=$ PtdIns, $\mathrm{C}=$ PtdSer; WT = wild-type (type sauvage) ; oil = régime alimentaire contrôle de mélange d'huiles; $\mathrm{Tg}=$ souris $\mathrm{Tg} 2576$; DHA = régime enrichi en DHA. Issu de C. Bascoul-Colombo, Ph.D. Thèse (2009) Cardiff University, avec autorisation.

méthodes de base - l'analyse de flux et l'analyse de contrôle métabolique (MCA, metabolic control analysis) - est bien décrite par Fell (Fell, 1997). En appliquant l'analyse de contrôle métabolique à plusieurs cultures agricoles clé - huile de palme (Ramli et al., 2002, 2009), soja (Guschina et al., 2014), olive (Ramli et al., 2002, 2005), colza (Weselake et al., 2008; Tang et al., 2012) - nous avons obtenu des données sur la 
Tableau 4. Valeurs de blocage du contrôle de flux pour différents oléagineux.

\begin{tabular}{cccc}
\hline & Synth. A.G. & Assemblage lipid. & Méthode \\
\hline Olive & 0,57 & 0,43 & SM \\
Huile de palme & $0,64 / 0,6$ & $0,36 / 0,39$ & SM/DM \\
Colza (Canola) & $0,31 / 0,27$ & $0,69 / 0,73$ & SM/DM \\
Tg Canola* & 0,49 & 0,51 & SM \\
Soja & $0,70 / 0,56$ & $0,30 / 0,44$ & SM/DM \\
\hline
\end{tabular}

* Contient une surexpression de DGAT1. SM, manipulation simple DM, double manipulation. Voir texte pour les références.

contribution relative des acides gras synthétisés et de l'assemblage des lipides au sein de la graine (Tab. 4). Les données ont montré que le contrôle du flux carbonique dans le triacylglycerol (TAG) des stocks de lipides était partagé entre les deux parties de la voie biosynthétique, mais avec davantage de contrôle de la synthèse d'acides gras dans trois des quatre cultures. Dans le colza, cependant, l'assemblage de lipides était plus important (Tab. 4). En fait, des expériences biochimiques précédentes avaient déjà indiqué que l'enzyme terminale de la voie de Kennedy (DGAT, diacylglycerol acyltransferase) avait un rôle significatif dans le contrôle du flux (Perry et Harwood, 1993; Perry et al., 1999). Donc, nous avons augmenté l'expression de DGAT 1 dans des lignées transgéniques et avons montré comment ceci a modifié l'équilibre du contrôle entre les deux blocs de réactions (Weselake et al., 2008). Quand ces graines transgéniques ont été plantées pour des essais de plein champ sur deux années successives, une augmentation de $8 \%$ du rendement en huile a été observée (Taylor et al., 2009). Ce résultat rassurant a montré l'utilité d'utiliser l'analyse de contrôle de flux pour sous-tendre des tentatives futures d'utiliser une modification génétique pour accroître les rendements en huile (Harwood et al., 2013).

Un point mérite d'être ajouté concernant la manipulation de la culture. La théorie derrière l'analyse de contrôle postule que toutes les étapes (enzymes) de la voie métabolique exercent un contrôle sur la production des lipides. Plus précisément, dans un ensemble de conditions données, une (ou plusieurs) enzymes limitantes seront plus importantes que d'autres dans le contrôle du flux lipidique, mais le changement d'une étape (comme nous l'avons fait avec DGAT) modifie la distribution si bien afin que d'autres enzymes exercent davantage de contrôle (voir le Tab. 4).

En prenant en compte ces données, il est important de considérer l'utilisation de constructions de gènes multiples. Cette approche a été plus particulièrement appliquée à la manipulation des acides gras pour permettre la production d'huiles végétales riches en AGPI n-3 à longue chaîne comme les huiles de poisson (Wu et al., 2005; Cheng et al., 2010; Ruiz-Lopez et al., 2013; Patric et al., 2012). Elle a commencé à être utilisée pour accroître les rendements en huile. En particulier, les stratégies 'push/pull' (augmentation de la synthèse d'AG/assemblage de TAG) ont été utilisé avec le gène Wrinkled 1 pour augmenter le flux de carbone dans la voie de DGAT, «poussant » les produits en TAG (Guschina et al., 2014; Vanherke et al., 2013). Il est probable que de telles «stratégies multi-cassettes » deviendront la norme dans l'avenir.

\section{Résumé}

Michel Chevreul (1786-1889) a continué de travailler dans son laboratoire et d'enseigner à ses étudiants à plus de 100 ans. Comme Bob Dylan le disait : "What's money? A man is a success if he gets up in the morning and goes to bed at night and in between does what he wants to do.» («Qu'est-ce que l'argent? Un homme a rencontré le succès s'il se lève le matin et se couche le soir et qu'entre les deux, il a fait ce qu'il voulait. »)

Inspiré par les lipides, j'ai énormément apprécié mes années d'enseignement et de recherche. Que pourrais-je demander de plus?

\section{L'auteur ne déclare aucun conflit d'intérêt.}

\section{Biographie}

John Harwood est professeur et directeur adjoint de l'École de biosciences de l'Université de Cardiff, au Royaume-Uni. Ses recherches concernent le métabolisme et la fonction des acyl-lipides et embrassent des aspects médicaux, la plante et le métabolisme algal et les mécanismes moléculaires de réponses au stress. Il est l'auteur de quatre livres, a été l'éditeur de 14 ouvrages et écrit plus de 600 publications scientifiques. Le travail de son laboratoire a été reconnu par de nombreuses distinctions comme celles de la Phytochemical Society of Europe, le Terry Galliard, le Kunio Yagi, l'ISF International et le Supelco-Nicholas Pelick. Son passe-temps est l'escalade et il a réalisé quelques 1200 premières ascensions sur les quatre continents.

\section{Références}

Avery SV, Lloyd D, Harwood JL. 1995a. Temperature-dependant changes in plasma membrane lipid order and the phagocytic activity of the amoeba Acanthamoeba castellanii are closely connected. Biochem. J. 312: 811-816.

Avery SV, Harwood JL, Lloyd D. 1995b. Quantification and characterization of phagocytosis in the soil amoeba Accanthamoeba castellenii by flow cytometry. Appl. Environ. Microbial. 61: 1124-1132.

Barber GA. 1963. The formation of uridine diphosphate L-rhamnose by enzymes of the tobacco leaf. Arch. Biochem. Biophys. 103: 276-282.

Bargerger-Gateau P, Letenneur L, Deschamps V, et al. 2002. Fish, meat and the risk of dementia: Cohort study. Br. Med. J. 325: 932-933.

Burns DD, Galliard T, Harwood JL. 1977. Catabolism of sulphoquinovosyldiacylglycerol by an enzyme preparation from Phaseolus multiflorus. Phytochemistry 16: 651-654.

Calder PC, Zurier RB. 2001. Polyunsaturated fatty acids and rheumatoid arthritis. Curr. Opin. Clin. Nutr. Metab. Care 4: 115-121.

Cheng B, Wu G, Vrinten P, et al. 2010. Towards the production of high levels of eicosapentaenoic acid in transgenic plants: The effects of different host species, genes and promoters. Transgenic Res. 19: 221-229. 
Clements JA. 1997. Lung surfactant: A personal perspective. Ann. Rev. Physiol. 59: 1-21.

Curtis CL, Rees SG, Cramp J, et al. 2002. Effects of n-3 fatty acids on cartilage metabolism. Proc. Nutri. Soc. 61: 381-389.

Dayan FE, Kagan IA, Rimando AM. 2003. Elucidation of the biosynthetic pathway of allelochemical sorgoleone using retrobiosynthetic NMR analysis. J. Biol. Chem. 278: 28607-28611.

Denger K, Weiss M, Felux A-K, et al. 2014. Sulphoglycolysis in Escherichia coli K-12 closes a gap in the biogeochemical sulphur cycle. Nature 507: 114-117.

Elder GA, Sosa G, De Gasperi R. 2010. Transgenic mouse models of Alzheimer's disease. Mt. Sinai J. Med. 77: 69-81.

Falinska AM, Bascoul-Colombo C, Guschina IA, Good M, Harwood JL. 2012. The role of n-3 dietary polyunsaturated fatty acids in brain function and amelioration of Alzheimer's disease: Opportunities for biotechnology in the development of nutraceuticals. Biocat. Agric. Biotechnol. 1: 159-166.

Fell DA. 1997. Understanding the control of metabolism. London: Portland Press.

Fenchel T. 1984. Ecology of Protozoa. Madison, Wis: Science Technology Publishers.

Frenchel T. 1982. Ecology of heterotrophic microflagellates. IV. Quantitative occurance and importance as bacterial consumers. Mar. Ecol. Prog. Ser. 9: 35-42.

Gunstone FD, Harwood JL, Dijkstra AJ (eds.). 2007. The lipid handbook, 3rd edn. Boca Raton, FL: Taylor and Francis.

Gurr MI, Robinson MP, James AT. 1969. The mechanism of formation of polyunsaturated fatty acids by photosynthetic tissues. Eur. J. Biochem. 9: 70-78.

Guschina IA, Everard JD, Kinney AJ, Quant PA, Harwood JL. 2014. Studies on the regulation of lipid biosynthesis in plants: Application of control analysis in soybean. Biochim. Biophys. Acta 1836: 1488-1500.

Harwood JL, in: Stumpf PK (ed.), Biochemistry of plants. New York: Academic Press, 1980, Vol. 4, pp. 301-320.

Harwood JL, Stumpf PK. 1971. Control of fatty acid synthesis in germinating seeds. Arch. Biochem. Biophys. 142: 281-291.

Harwood JL, Caterson B. 2006. Dietary omega-3 polyunsaturated fatty acids and inflammation. Lipid Technol. 18: 7-10.

Harwood JL, Stumpf PK. 1972. Palmitic and stearic acid synthesis by an avocado supernatant system. Arch. Biochem. Biophys. 148: 282-290.

Harwood JL, Desai R, Hext P, Tetley T, Richards RJ. 1975. Characterisation of pulmonary surfactant from ox, rabbit, rat and sheep. Biochem. J. 151: 707-714.

Harwood JL, Ramli US, Tang M, et al. 2013. Regulation and enhancement of lipid accumulation in oil crops: The use of metabolic control analysis for informed genetic manipulation. Eur. J. Lipid Sci. Technol. 364: 393-401.

Hurst S, Rees SG, Randerson PF, Caterson B, Harwood JL. 2009. Contrasting effects of n-3 and n-6 fatty acids on cyclooxygenase2 in model systems for arthritis. Lipids 44: 889-896.

Jackowski S, Rock CO. 1987. Acetoacetyl-acyl carrier protein synthase, a potential regulator of fatty acid synthesis in bacteria. $J$. Biol. Chem. 262: 7927-7931.

Jones AL, Pruitt NL, Lloyd D, Harwood JL. 1991. Temperature induced changes in the synthesis of unsaturated fatty acids by Acanthamoeba castellanii. J. Protozol. 38: 532-536.
Jones AL, Lloyd D, Harwood JL, 1993. Rapid induction of microsomal $\Delta 12$ (w6)-desaturase activity in chilled Acanthamoeba castellanii. Biochem. J. 1993, 296, 183-188.

Jones AL, Gane AM, Herbert D, et al. 2003. $\beta$-Ketoacyl-acyl carrier protein synthase III from pea: Properties, inhibition by a novel thiolactomycin analogue and isolation of cDNA clone encoding the enzyme. Planta 216: 752-761.

Kalmijn S, Launer L, Ott A, et al. 1997. Dietary fat intake and the risk of incident dementia in the Rotterdam study. Ann. Neurol. 42: 776-782.

Kikukawa H, Sakuradani E, Kishino S, et al. 2013. Characterization of a trifunctional fatty acid desaturase from oleaginous filamentous fungus Martierella alpine 1S-4 using a yeast expression system. J. Biosci. Bioeng. 116: 672-676.

Lands WEM. 2014. Historical perspectives on the impact of n-3 and n-6 nutrients on health. Prog. Lipid Res. 55: 17-29.

Lee RF, Benson AA. 1964. The metabolism of glyceryl [35S]sulphoquinovoside by the coral tree, Erythrina cristagalli and alfalfa Medicago sativa. Biochim. Biophys. Acta 261: 35-37.

Lehmann J, Benson AA. 1964. The plant sulfolipids. Sulfosugar synthesis from methyl hexoseenides. J. Am. Chem. Soc. 86: 44694472.

Martelli H, Benson AA. 1964. Sulphocarbohydrate metabolism. 1. Bacterial production and utilization of sulphoacetate. Biochim. Biophys. Acta 93: 169-171.

McCann JC, Ames BN. 2005. Is docosahexaenoic acid, an n-3 longchain polyunsaturated fatty acid, required for development of normal brain function? An overview of evidence from cognitive and behavioural tests in humans and animals. Am. J. Clin. Nutr. 82: 281-295.

Morris MC, Evans DA, Bienias JL, et al. 2003. Consumption of fish and n-3 fatty acids and the risk of incident Alzheimer's disease. Arch. Neurol. 60: 940-946.

Nichols BW, James AT, Breuer J. 1967. Interrelationships between fatty acid biosynthesis and acyl-lipid synthesis in Chlorella vulgaris. Biochem. J. 104: 486-496.

Patric JR, Shrestha P, Zhou XR, et al. 2012. Metabolic engineering plant seeds with fish oil-like levels of DHA. PLos One 7: e49165.

Perry HJ, Bligny R, Gout E, Harwood JL. 1999. Changes in Kennedy pathway intermediates associated with increased triacylglycerol synthesis in oil-seed rape. Phytochemistry 52: 799-804.

Perry HJ, Harwood JL. 1993. Radiolabelling studies of acyl lipids in developing seeds of Brassica napus: Use of [1-14C]acetate precursor. Phytochemistry 33: 329-353.

Pugh CE, Roy AB, Hawkes T, Harwood JL. 1995. A new pathway for the synthesis of the plant sulpholipid, sulphoquinovosyldiacylglycerol. Biochem. J. 309: 513-519.

Ramli US, Baker DS, Quant PA, Harwood JL. 2002. Control analysis of lipid biosynthesis in tissue cultures of oil crops shows that flux control is shared between fatty acid synthesis and lipid assembly. Biochem. J. 364: 393-401.

Ramli US, Salas JJ, Quant PA, Harwood JL. 2005. Metabolic control analysis reveals an important role for diacylglycerol acyltransferase in olive but not in oil palm lipid accumulation. FEBS J. 272: 5764-5770. 
Ramli US, Salas JJ, Quant PA, Harwood JL. 2009. Use of metabolic control analysis to give quantitative information on the control of lipid biosynthesis in the important oil crop, Elaeis guineensis (oil palm). New Phytol. 184: 330-339.

Robertson B, Van Golde LMG, Batenburg JJ, eds. 1984. Pulmonary Surfactant, Amsterdam: Elsevier.

Roy AB, Hewlins MJE, Ellis AJ, Harwood JL, White, GF. 2003. Glycolyic breakdown of sulphoquinovose in bacteria: A missing link in the sulphur cycle. Appl. Environ. Microbiol. 69: 64346441.

Ruiz-Lopez N, Haslam RP, Usher SL, et al. 2013. Reconstitution of EPA and DHA biosynthesis in Arabidopsis. Iterative metabolic engineering for the synthesis of n-3 LC-PUFAs in transgenic plants. Metab. Eng. 17: 30-41.

Sayanova O, Haslam R, Guschina I, et al. 2006. A bi-functional $\Delta 12$, 415 -desaturase from Accanthamoeba castellanii directs the synthesis of highly unusual n-1 series unsaturated fatty acids. J. Biol. Chem. 281: 36533-36541.

Shimijima M. 2011. Biosynthesis and functions of the plant sulpholipid. Prog. Lipid Res. 50: 234-239.

Slack CR, Roughan PG, Browse J. 1979. Evidence for an oleoylphosphatidylcholine desaturase in microsomal preparations from cotyledons of sunflower seeds. Biochem. J. 179: 649-652.

Tang M, Guschina IA, O'Hara P, et al. 2012. Metabolic control analysis of developing oilseed rape embryos shows that lipid assembly exerts significant control over oil accumulation. New Phytologist 196: 415-426.
Taylor DC, Zhang Y, Kumar A, et al. 2009. Molecular modification of triacylglycerol accumulation by over-expression of DGAT1 to produce canola with increased seed oil content under field conditions. Botany 87: 533-543.

Vanherke T, EI Tahchy A, Shrestha P, et al. 2013. Synergistic effect of WRI 1, DGAT 1 coexpression on triacylglycerol biosynthesis in plants. FEBS Lett. 587: 364-369.

Wallis JG, Browse J. 2002. Mutants of Arabidopsis reveal many roles for membrane lipids. Prog. Lipid Res. 41: 254-278.

Walsh MC, Klopfenstein WE, Harwood JL. 1990. The short chain condensing enzyme has a widespread occurance in the fatty acid synthases of higher plants. Phytochemistry 29: 3797-3799.

Watson RR, ed. 2009. Fatty Acids in Health Promotion and Disease Causation, Urbana, IL: AOCS Press.

Weselake RJ, Shah S, Tang M, et al. 2008. Metabolic control analysis is helpful for informed genetic manipulation of oilseed rape (Brassica napus) to increase seed oil content. J. Exptl. Botany 59: 3543-3549.

Wharfe J, Harwood JL. 1978. Fatty acid biosynthesis in the leaves of barley, wheat and pea. Biochem. J. 174: 163-169.

Wrobel S. 2004. Bubbles, babies and biology: The story of surfactant. FASEB J. 18: 1624e.

Wu G, Traksa M, Datla N, et al., 2005. Stepwise engineering to produce high yields of very long-chain polyunsaturated fatty acids in plants. Nat. Biotechnol. 23: 1013-1017.

Zainal Z, Longman AJ, Hurst S, et al. 2009. Relative efficacies of omega-3 polyunsaturated fatty acids in reducing expression of key proteins in a model system for studying osteoarthritis. Osteoarthritis Cartilage 17: 882-891.

Cite this article as: John L. Harwood, Traduction de Valérie Duflot et Philippe Guesnet, $O C L$ journal. Inspiré par les lipides (Médaille Chevreul 2014). OCL 2015, 22(2) A202. 